\title{
Front Matter: Volume 7578
}

, "Front Matter: Volume 7578," Proc. SPIE 7578, Solid State Lasers XIX:

Technology and Devices, 757801 (17 March 2010); doi: 10.1117/12.855211

SPIE. Event: SPIE LASE, 2010, San Francisco, California, United States 


\title{
PROCEEDINGS OF SPIE
}

\section{Solid State Lasers XIX: Technology and Devices}

\author{
W. Andrew Clarkson \\ Norman Hodgson \\ Ramesh K. Shori \\ Editors
}

24-28 January 2010

San Francisco, California, United States

Sponsored and Published by

SPIE

Volume 7578 
The papers included in this volume were part of the technical conference cited on the cover and title page. Papers were selected and subject to review by the editors and conference program committee. Some conference presentations may not be available for publication. The papers published in these proceedings reflect the work and thoughts of the authors and are published herein as submitted. The publisher is not responsible for the validity of the information or for any outcomes resulting from reliance thereon.

Please use the following format to cite material from this book:

Author(s), "Title of Paper," in Solid State Lasers XIX: Technology and Devices, edited by

W. Andrew Clarkson, Norman Hodgson, Ramesh K. Shori, Proceedings of SPIE Vol. 7578 (SPIE, Bellingham, WA, 2010) Article CID Number.

ISSN 0277-786X

ISBN 9780819479747

Published by

SPIE

P.O. Box 10, Bellingham, Washington 98227-0010 USA

Telephone +1 3606763290 (Pacific Time) · Fax +1 3606471445

SPIE.org

Copyright (C) 2010, Society of Photo-Optical Instrumentation Engineers

Copying of material in this book for internal or personal use, or for the internal or personal use of specific clients, beyond the fair use provisions granted by the U.S. Copyright Law is authorized by SPIE subject to payment of copying fees. The Transactional Reporting Service base fee for this volume is $\$ 18.00$ per article (or portion thereof), which should be paid directly to the Copyright Clearance Center (CCC), 222 Rosewood Drive, Danvers, MA 01923. Payment may also be made electronically through CCC Online at copyright.com. Other copying for republication, resale, advertising or promotion, or any form of systematic or multiple reproduction of any material in this book is prohibited except with permission in writing from the publisher. The CCC fee code is $0277-786 \mathrm{X} / 10 / \$ 18.00$.

Printed in the United States of America.

Publication of record for individual papers is online in the SPIE Digital Library.

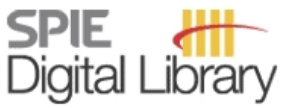

SPIEDigitalLibrary.org

Paper Numbering: Proceedings of SPIE follow an e-First publication model, with papers published first online and then in print and on CD-ROM. Papers are published as they are submitted and meet publication criteria. A unique, consistent, permanent citation identifier (CID) number is assigned to each article at the time of the first publication. Utilization of CIDs allows articles to be fully citable as soon they are published online, and connects the same identifier to all online, print, and electronic versions of the publication. SPIE uses a six-digit CID article numbering system in which:

- The first four digits correspond to the SPIE volume number.

- The last two digits indicate publication order within the volume using a Base 36 numbering system employing both numerals and letters. These two-number sets start with 00, 01, 02, 03, 04, $05,06,07,08,09,0 A, 0 B \ldots 0 Z$, followed by 10-1Z, 20-2Z, etc.

The CID number appears on each page of the manuscript. The complete citation is used on the first page, and an abbreviated version on subsequent pages. Numbers in the index correspond to the last two digits of the six-digit CID number. 


\section{Contents}

xi

$\mathrm{XV}$

Ultrafast fiber laser technology: status and prospects (Plenary Paper) [7579-102]

A. Tünnermann, J. Limpert, Friedrich-Schiller-Univ. Jena (Germany) and Fraunhofer-Institute for Applied Optics and Precision Engineering (Germany)

\section{SESSION 1 SPACE-QUALIFIED LASERS I}

757802 Spaceborne laser instruments for high-resolution mapping (Invited Paper) [7578-01]

A. W. Yu, M. A. Krainak, D. J. Harding, J. B. Abshire, X. Sun, S. Valett, J. Cavanaugh,

L. Ramos-Izquierdo, NASA Goddard Space Flight Ctr. (United States)

757805 Quasi-CW laser diode arrays for space applications [7578-04]

A. Seas, NASA Goddard Space Flight Ctr. (United States); E. Troupaki, A. A. Vasilyev,

H. Conley, Sigma Space Corp. (United States)

\section{SESSION 2 SPACE-QUALIFIED LASERS II}

$757806 \quad$ Extended testing of laser systems for space applications [7578-06]

A. Seas, A. W. YU, NASA Goddard Space Flight Ctr. (United States); A. Melak, D. Mostofi,

E. Troupaki, A. Vasilyev, H. Conley, Sigma Space Corp. (United States)

757808 A highly reliable single mode laser for space application [7578-08]

S. X. Li, NASA Goddard Space Flight Ctr. (United States); A. A. Vasilyev, Sigma Space Corp. (United States); M. A. Stephen, A. W. YU, M. A. Krainak, G. B. Shaw, NASA Goddard Space

Flight Ctr. (United States); A. A. Rosanova, T. I. Filemyr, Bastion Technologies, Inc. (United States)

757809 Space laser transmitter development for ICESat-2 mission [7578-09]

A. W. Yu, M. A. Stephen, S. X. Li, G. B. Shaw, A. Seas, E. Dowdye, NASA Goddard Space Flight Ctr. (United States); E. Troupaki, P. Liiva, Sigma Space Corp. (United States); D. Poulios, American Univ. (United States); K. Mascetti, Orbital Sciences Corp. (United States)

\section{SESSION 3 DISK LASERS I}

7578 OA 700W intracavity-frequency doubled Yb:YAG thin-disk laser at $100 \mathrm{kHz}$ repetition rate (Invited Paper) [7578-10]

C. Stolzenburg, W. Schüle, I. Zawischa, A. Killi, D. Sutter, TRUMPF Laser GmbH \& Co. KG (Germany)

7578 OB Ytterbium-based disk amplifier for an ultra-short pulse laser [7578-1 1]

J. Vetrovec, D. A. Copeland, Aqwest LLC (United States); D. Du, B. Schmidt, General Atomics Aeronautical Systems, Inc. (United States) 
7578 OC Latest advances in high-power disk lasers [7578-12]

D. Havrilla, TRUMPF Inc. (United States); R. Brockmann, TRUMPF Laser GmbH \& Co. KG

(Germany)

7578 OD A design for a 100-kW rotary disk laser oscillator with good beam quality [7578-13]

S. Basu, Sparkle Optics Corp. (United States)

$7578 \mathrm{OE} \mathrm{CW}$ laser operation of a highly-doped $\mathrm{Tm}: \mathrm{KLu}\left(\mathrm{WO}_{4}\right)_{2} / \mathrm{KLu}\left(\mathrm{WO}_{4}\right)_{2}$ thin disk epitaxial laser [7578-14]

S. Vatnik, I. Vedin, Institute of Laser Physics (Russian Federation); M. C. Pujol, X. Mateos, J. J. Carvajal, M. Aguilo, F. Diaz, Univ. Rovira i Virgili (Spain); U. Griebner, V. Petrov, Max-Born-Institut für Nichtlineare Optik und Kurzzeitspektroskopie (Germany)

SESSION 4

DISK LASERS II

7578 OF Improved bond strength characterization of chemically activated direct bonding (CADB) epoxy-free bonded solid state laser materials (Invited Paper) [7578-15]

N. G. Traggis, N. R. Claussen, Precision Photonics Corp. (United States)

7578 OG Comparative performance of ASE suppressed ceramic Yb:YAG thin disks [7578-16]

D. Bossert, P. Avizonis, Boeing-SVS, Inc. (United States); A. Killi, TRUMPF Laser GmbH \& Co. KG (Germany)

7578 Ol Thermal effect of cryogenic Yb:YAG total-reflection active-mirror laser [7578-18] H. Furuse, Institute for Laser Technology (Japan); J. Kawanaka, N. Miyanaga, Osaka Univ. (Japan); T. Saiki, M. Fujita, K. Imasaki, Institute for Laser Technology (Japan); K. Takeshita, S. Ishii, Mitsubishi Heavy Industries, Ltd. (Japan); Y. Izawa, Institute for Laser Technology (Japan)

\section{SESSION 5 SLAB AND WAVEGUIDE LASERS}

7578 OK 400W Yb:YAG planar waveguide laser using novel unstable resonators [7578-20] I. J. Thomson, H. J. Baker, K. Wlodarczyk, N. Trela, D. R. Hall, Heriot-Watt Univ. (United Kingdom)

7578 OL Power-scaling Nd:YAG's quasi-four-level transition [7578-21]

J. I. Mackenzie, Univ. of Southampton (United Kingdom)

7578 ON Recent progresses in INNOSLAB lasers and their harmonic generation [7578-23]

D. Li, S. Fu, X. Liu, P. Shi, J. Chen, A. Shell, B. Qi, C.-R. Haas, J. Wang, K. Du, EdgeWave GmbH (Germany)

SESSION $6 \quad$ VISIBLE AND UV LASERS I: JOINT SESSION WITH CONFERENCES 7580 AND 7582

757800 Efficient, green laser based on a blue-diode pumped rare-earth-doped fluoride crystal in an extremely short resonator [7578-24]

M. Strotkamp, T. Schwarz, B. Jungbluth, H. Faidel, M. Leers, Fraunhofer-Institut für Lasertechnik (Germany) 
7578 OP Frequency doubling of fiber laser radiation of large spectral bandwidths [7578-25]

S. Nyga, J. Geiger, B. Jungbluth, Fraunhofer-Institut für Lasertechnik (Germany)

\section{SESSION $7 \quad$ VISIBLE AND UV LASERS II}

$75780 Q \quad$ A $200 \mathrm{~mW}, \mathrm{CW}, 355 \mathrm{~nm}$ laser based on DPSS side pumped, internally frequency tripled technology [7578-92]

S. M. Jarrett, G. P. Shellikeri, O. Varela, DPSS Lasers Inc. (United States)

7578 OR Compact and efficient continuous wave UV DPSS laser [7578-26]

J. Hellström, G. Elgcrona, E. Illy, H. Karlsson, Cobolt AB (Sweden)

7578 OS Low-cost frequency-converted laser light sources [7578-27]

J. Konttinen, P. Tuomisto, T. Vallius, P. Pietilä, T. Jouhti, EpiCrystals, Inc. (Finland)

7578 OT Half-Watt single frequency yellow $561 \mathrm{~nm}$ and yellow-green $553 \mathrm{~nm}$ DPSS lasers with record $19 \%$ optical conversion efficiency [7578-28]

T. Georges, C. Chauzat, A. Poivre, Oxxius SA (France)

$7578 \mathrm{OU}$ Short and long term frequency stability of linear monolithic intra-cavity frequency-doubled solid-state laser [7578-29]

T. Georges, C. Chauzat, A. Poivre, N. Landru, J. Rouvillain, Oxxius SA (France)

7578 OV Frequency doubled pulsed single longitudinal mode Nd:YAG laser at $1319 \mathrm{~nm}$ with pulse build-up negative feedback controls [7578-30]

R. Bakanas, J. Pileckas, Geola Digital UAB (Lithuania)

\section{SESSION 8 OPS LASERS I}

7578 OW Ten years optically pumped semiconductor lasers: review, state-of-the-art, and future developments [7578-31]

C. Kannengiesser, V. Ostroumov, V. Pfeufer, W. Seelert, C. Simon, R. von Elm, A. Zuck, Coherent Lubeck GmbH (Germany)

$75780 \mathrm{X}$ GaSb-based optically pumped semiconductor disk lasers emitting in the $\mathbf{2 . 0 - 2 . 8} \boldsymbol{\mu m}$ wavelength range (Invited Paper) [7578-32]

B. Rösener, M. Rattunde, R. Moser, S. Kaspar, C. Manz, K. Köhler, J. Wagner, FraunhoferInstitut für Angewandte Festkörperphysik (Germany)

7578 OY 200-nm tunable fiber vertical-cavity surface emitting laser [7578-33]

S. L. Vetter, N. Laurand, Univ. of Strathclyde (United Kingdom); M. Guina, Tampere Univ. of Technology (Finland); M. D. Dawson, S. Calvez, Univ. of Strathclyde (United Kingdom)

$75780 Z$ Low-temperature study of lasing characteristics for 1.3pumped by an actively Q-switched Nd:YAG laser [7578-34]

K. W. Su, Y.-F. Chen, S. C. Huang, A. Li, S. C. Liu, Y. F. Chen, K. F. Huang, National Chiao Tung Univ. (Taiwan) 
757811 A 7-W 1178nm GalnNAs based disk laser for guide star applications [7578-36]

T. Leinonen, V.-M. Korpijärvi, J. Puustinen, A. Härkönen, M. Guina, M. Pessa, Tampere Univ. of Technology (Finland)

$757812 \quad 532 \mathrm{~nm}$ laser sources based on intracavity frequency doubling of multi-edge-emitting diode lasers [7578-37]

K. Li, N. J. Copner, Univ. of Glamorgan (United Kingdom); C. B. E. Gawith, Covesion Ltd. (United Kingdom); I. G. Knight, Bookham Technology plc (United Kingdom); H.-U. Pfeiffer, Bookham (Switzerland) AG (Switzerland); B. Musk, Gooch \& Housego, Torquay (United Kingdom)

\section{SESSION 10 CERAMIC MATERIALS AND THERMAL MANAGEMENT}

757815 Yb:YAG composite ceramic laser [7578-39]

E. Pawlowski, M. Kluge, Y. Menke, U. Peuchert, A. Engel, SCHOTT AG (Germany); Y. L. Aung, A. Ikesue, World Lab Co., Ltd. (Japan); K. Beil, R. Peters, H. Kuehn, K. Petermann, Univ. Hamburg (Germany); P. Heist, JENOPTIK Laser, Optik, Systeme GmbH (Germany)

757816 Thermally induced aberrations in solid-state lasers [7578-41]

M. M. Tilleman, Elbit Systems of America (United States)

\section{SESSION 11 ULTRAFAST LASERS}

757817 Efficient, high power nonlinear mirror modelocking of a bounce geometry laser [7578-93] G. M. Thomas, S. P. Chard, M. J. Damzen, Imperial College London (United Kingdom)

757818 Multi-kHz, multi-mJ, phase stabilized, OPCPA amplifier system [7578-43] M. Hemmer, A. Vaupel, B. Webb, M. Richardson, CREOL, The College of Optics and Photonics, Univ. of Central Florida (United States)

75781 A Sectional chirped volume Bragg grating compressors for high-power chirped-pulse amplification [7578-45]

O. Andrusyak, CREOL, The College of Optics and Photonics, Univ. of Central Florida (United States); L. Canioni, Univ. Bordeaux 1 (France); I. Cohanoshi, OptiGrate Corp. (United States); M. Delaigue, Amplitude Systemes (United States); E. Rotari, V. Smirnov, OptiGrate Corp. (United States); L. Glebov, CREOL, The College of Optics and Photonics, Univ. of Central Florida (United States)

7578 IB A compact dispersive delay generator using angular dispersion amplification [7578-46] S. Basu, Sparkle Optics Corp. (United States)

7578 1C Time-gating processes in intra-cavity mode-locking devices like saturable absorbers and Kerr cells [7578-47]

N. S. Prasad, NASA Langley Research Ctr. (United States); C. Roychoudhuri, Univ. of Connecticut (United States) 
7578 1D Q-switched resonantly diode-pumped Er:YAG laser [7578-48]

I. Kudryashov, A. Katsnelson, Princeton Lightwave, Inc. (United States)

7578 1E Efficient, 1.5W CW and 7mJ quasi-CW TEMoo mode operation of a compact diode-

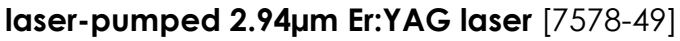

J. G. Sousa, Sheaumann Laser, Inc. (United States); D. Welford, Endeavour Laser

Technologies (United States); J. Foster, Sheaumann Laser, Inc. (United States)

7578 IF Two-micron cryogenically-cooled solid-state lasers: recent progress and future prospects [7578-50]

J. I. Mackenzie, J. W. Kim, L. Pearson, W. O. S. Bailey, Y. Yang, W. A. Clarkson, Univ. of Southampton (United Kingdom)

7578 1G Atmospheric propagation testing with a high power, tunable thulium fiber laser system [7578-51]

T. S. McComb, L. Shah, R. A. Sims, C. C. C. Willis, P. Kadwani, V. Sudesh, M. Richardson, Townes Laser Institute, CREOL, The College of Optics and Photonics, Univ. of Central Florida (United States)

\section{SESSION 13 MID-INFRARED LASERS II}

7578 1H Optically dense Fe:ZnSe crystals for energy scaled gain switched lasing [7578-52] N. Myoung, V. V. Fedorov, S. B. Mirov, The Univ. of Alabama at Birmingham (United States)

757811 Laser-spectroscopic study of Er doped $\mathrm{PbWO}_{4}$ as laser and stimulated Raman scattering active crystals [7578-53]

I. Mirov, Univ. of Richmond (United States) and The Univ. of Alabama at Birmingham (United States); V. V. Fedorov, The Univ. of Alabama at Birmingham (United States)

$75781 \mathrm{~J}$ Cr-ZnSe passively Q-switched fiber-bulk Ho:YAG hybrid laser [7578-54]

Y. Terekhov, I. S. Moskalev, D. V. Martyshkin, V. V. Fedorov, The Univ. of Alabama at Birmingham (United States); S. B. Mirov, The Univ. of Alabama at Birmingham (United States) and Photonics Innovations, Inc. (United States)

7578 1K InP diode-pumped Cr:ZnS and Cr:ZnSe highly efficient, widely tunable, mid-IR lasers [7578-55]

I. S. Moskalev, V. V. Fedorov, The Univ. of Alabama at Birmingham (United States); S. B. Mirov, The Univ. of Alabama at Birmingham (United States) and Photonics Innovations, Inc. (United States)

7578 IL $\quad \mathrm{Cr}^{2+}: Z n S e$ master oscillator/power amplifier for improved power scaling [7578-94]

P. A. Berry, K. L. Schepler, Air Force Research Lab. (United States)

\section{SESSION 14 Q-SWITCHED LASERS}

$75781 \mathrm{M}$ Physical and engineering aspects of passively Q-switched microlasers (Invited Paper) [7578-56]

Y. Kalisky, Nuclear Research Ctr. Negev (Israel) 
7578 IN An injection seeded single frequency Nd:YAG Q-switched laser with precisely controllable laser pulse firing time [7578-57]

F. F. Wu, A. Khizhnyak, V. Markov, MetroLaser, Inc. (United States)

$7578102 \mathrm{MHz}$ repetition rate, 200ps pulse duration from a monolithic passively Q-switched microchip laser [7578-58]

A. Steinmetz, D. Nodop, J. Limpert, Friedrich-Schiller-Univ. Jena (Germany); R. Hohmuth,

W. Richter, BATOP GmbH (Germany); A. Tünnermann, Friedrich-Schiller-Univ. Jena

(Germany)

7578 IP Intra-cavity frequency doubled Nd:YAG laser with dual-stability-range cavity emitting high-power near-diffraction-limited radiation in CW and Q-switched mode [7578-59]

D. Woll, J. Gregg, J. Lefort, J. J. Morehead, J. Lindahl, JDSU (United States)

$75781 Q$ Reduction in timing jitter for a Cr:YAG Q-switched Nd:YAG laser [7578-60]

B. Cole, J. Lei, T. DiLazaro, B. Schilling, W. Trussell, L. Goldberg, US Army RDECOM CERDEC

(United States)

7578 IR Comparison of small fiber connectors for high-power transmission [7578-61]

S. Campbell, O. Blomster, M. Pålsson, Optoskand AB (Sweden)

7578 is Ultrashort pulse long distance fiber delivery [7578-62]

T. Le, G. Tempea, M. Hofer, Z. Cheng, A. Stingl, Femtolasers Produktions GmbH (Austria)

7578 iT Compact hollow fiber compression scheme for multi-mJ pulse generation [7578-63] A. Anderson, G. Tempea, M. Hofer, T. Prikoszovits, Z. Cheng, T. Le, A. Assion, Femtolasers Produktions $\mathrm{GmbH}$ (Austria)

$75781 \mathrm{U}$ Faraday isolators for high average power fundamental mode radiation [7578-64] K. Nicklaus, T. Langer, JT Optical Engine GmbH + Co. KG (Germany)

7578 IV Deterministic single shot and multiple shots bulk damage thresholds for doped and undoped crystalline and ceramic YAG [7578-65]

B. T. Do, Sandia National Labs. (United States); A. V. Smith, AS-Photonics, LLC (United States)

\section{SESSION 16 APPLICATIONS}

$75781 X \quad$ New developments in STED microscopy [7578-67]

A. Giske, J. Sieber, H. Gugel, M. Dyba, V. Seyfried, D. Gnass, Leica Microsystems CMS (Germany)

7578 IY Laser perforation of aluminum alloy sheet [7578-68]

L. Migliore, G. Nazary, Coherent, Inc. (United States)

$757812 \quad$ Enhanced productivity with high power short and ultrashort thin disk lasers [7578-69] J. Stollhof, TRUMPF Inc. (United States); S. Weiler, U. Stute, S. Massa, S. Buettner, B. Faisst, TRUMPF Laser GmbH \& Co. KG (Germany) 
$757821 \quad$ Applications of INNOSLAB lasers with tailored beam profiles [7578-71]

K. Du, P. Shi, A. Schell, D. Li, C.-R. Haas, S. Fu, EdgeWave GmbH (Germany)

POSTER SESSION

757822 Very compact and high-power CW self-Raman laser for ophthalmological applications [7578-72]

T. A. Ortega, Opto Eletrônica S.A. (Brazil) and Univ. of São Paulo (Brazil); A. D. Mota, G. Rossi, G. C. de Castro, Y. C. Fontes, G. Z. Costal, F. M. M. Yasuoka, M. A. Stefani, Opto Eletrônica S.A. (Brazil); A. Lee, H. Pask, Macquarie Univ. (Australia); J. C. de Castro N., Opto Eletrônica S.A. (Brazil) and Univ. of São Paulo (Brazil)

757824 Quasi-continuously pumped passively mode-locked $2.4 \%$ doped Nd:YAG oscillator-amplifier system in a bounce geometry [7578-74]

M. Jelínek, V. Kubeček, M. Čech, P. Hiršl, Czech Technical Univ. in Prague (Czech Republic)

757825 Four micron radiation generated by dysprosium doped lead thiogallate laser [7578-75] M. E. Doroshenko, T. T. Basiev, V. V. Osiko, General Physics Institute (Russian Federation); H. Jelínková, J. Šulc, M. Jelinek, M. Fibrich, Czech Technical Univ. in Prague (Czech Republic); V. V. Badikov, Kuban State Univ. (Russian Federation)

757826 Cr:ZnSe laser pumped with Tm:YAP microchip laser [7578-76]

P. Koranda, J. Šulc, Czech Technical Univ. in Prague (Czech Republic); M. Doroshenko, General Physics Institute (Russian Federation); H. Jelínková, Czech Technical Univ. in Prague (Czech Republic); T. T. Basiev, V. Osiko, General Physics Institute (Russian Federation); V. V. Badikov, D. Badikov, Kuban State Univ. (Russian Federation)

757827 Complex behavior of a $\mathrm{Yb}: \mathrm{GdVO}_{4}$ laser with bistability and polarization switching [7578-77] J. Liu, W. Han, Qingdao Univ. (China); H. Zhang, Shandong Univ. (China); H. Yang, Qingdao Univ. (China); V. Petrov, Max-Born-Institute for Nonlinear Optics and Ultrafast Spectroscopy (Germany)

757828 Pr:YAlO 3 and Pr:LiYF 4 laser emission comparison under GaN laser diode pumping [7578-78] M. Fibrich, H. Jelínková, J. Šulc, Czech Technical Univ. in Prague (Czech Republic); K. Nejezchleb, V. Škoda, Crytur Ltd. (Czech Republic)

7578 2A Pump laser effect on temporal jittering of pulses from passively Q-switched $\mathrm{Nd}: \mathrm{YVO}_{4}$ laser [7578-80]

K. Lee, Y. Kim, J. H. Kwon, J. Yi, Yeungnam Univ. (Korea, Republic of)

7578 2C Coherently pumped Er:YAlO 3 lasers [7578-82]

M. Nĕmec, H. Jelínková, J. Šulc, Czech Technical Univ. in Prague (Czech Republic);

K. Nejezchleb, V. Škoda, Cryłur Ltd. (Czech Republic)

$75782 \mathrm{E}$ Influence of temperature on Nd:YAG/V:YAG compact laser generation at $1444 \mathrm{~nm}$ [7578-84]

J. Šulc, J. Novák, H. Jelínková, Czech Technical Univ. in Prague (Czech Republic);

K. Nejezchleb, V. Škoda, Cryłur Ltd. (Czech Republic) 
$75782 \mathrm{~F} \quad$ Powerful narrow-line source of blue light for laser cooling $\mathrm{Yb} / \mathrm{Er}$ and Dysprosium atoms [7578-86]

S. Kobtsev, Novosibirsk State Univ. (Russian Federation) and Tekhnoscan JSC (Russian Federation); B. Lev, Univ. of Illinois at Urbana-Champaign (United States); J. Fortágh, Univ. of Tuebingen (Germany); V. Baraulya, Tekhnoscan JSC (Russian Federation)

$75782 \mathrm{G} \quad$ LIBS system with compact fiber spectrometer, head mounted spectra display and hand held eye-safe erbium glass laser gun [7578-87]

M. J. Myers, J. D. Myers, J. T. Sarracino, C. R. Hardy, B. Guo, S. M. Christian, J. A. Myers, F. Roth, A. G. Myers, Kigre, Inc. (United States)

7578 2H Dynamic 3D modeling of solid state laser resonators using a coupled thermo-optical finite element analysis [7578-88]

M. Wohlmuth, Friedrich-Alexander-Univ. Erlangen-Nürnberg (Germany); K. Altmann, LAS-CAD GmbH (Germany); J. Werner, C. Pflaum, Friedrich-Alexander-Univ. ErlangenNürnberg (Germany)

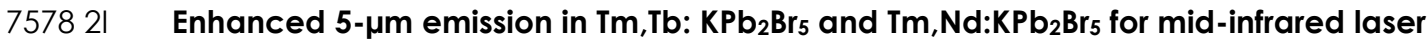
applications [7578-89]

A. G. Bluiett, D. Peele, K. Norman, Elizabeth City State Univ. (United States); E. Brown, U. Hömmerich, Hampton Univ. (United States); S. B. Trivedi, Brimrose Corp. of America (United States); J. M. Zavada, North Carolina State Univ. (United States)

7578 2J Single frequency monolithic green DPSS laser [7578-90]

J. Z. Sotor, A. J. Antończak, K. M. Abramski, Wroclaw Univ. of Technology (Poland)

Author Index 


\title{
Conference Committee
}

\author{
Symposium Chairs
}

Donald J. Harter, IMRA America, Inc. (United States)

Peter R. Herman, University of Toronto (Canada)

Symposium Cochairs

Alberto Piqué, Naval Research Laboratory (United States)

Friedhelm Dorsch, TRUMPF Photonics (United States)

Program Track Chair

Gregory J. Quarles, VLOC (United States)

Conference Chairs

W. Andrew Clarkson, University of Southampton (United Kingdom)

Norman Hodgson, Coherent, Inc. (United States)

Ramesh K. Shori, Naval Air Warfare Center (United States)

Program Committee

Santanu Basu, Sparkle Optics Corporation (United States)

Martin D. Dawson, University of Strathclyde (United Kingdom)

Adolf Giesen, Deutsches Zentrum für Luft- und Raumfahrt e.V. (Germany)

Hans-Dieter Hoffmann, Fraunhofer-Institut für Lasertechnik (Germany)

Helena Jelínková, Czech Technical University in Prague (Czech Republic)

Jacob I. Mackenzie, University of Southampton (United Kingdom)

Michio Oka, Sony Corporation (Japan)

Alan B. Petersen, Newport Spectra-Physics (United States)

Narasimha S. Prasad, NASA Langley Research Center (United States)

Martin C. Richardson, CREOL, The College of Optics and Photonics, University of Central Florida (United States)

Wolf Seelert, Coherent Lubeck GmbH (Germany)

Irina T. Sorokina, Norwegian University of Science and Technology (Norway)

David H. Titterton, Defence Science and Technology Laboratory

(United Kingdom) 
Session Chairs

1 Space-Qualified Lasers I

Narasimha S. Prasad, NASA Langley Research Center (United States)

2 Space-Qualified Lasers II

David H. Titterton, Defence Science and Technology Laboratory

(United Kingdom)

3 Disk Lasers I

Hans-Dieter Hoffmann, Fraunhofer-Institut für Lasertechnik (Germany)

4 Disk Lasers II

Santanu Basu, Sparkle Optics Corporation (United States)

$5 \quad$ Slab and Waveguide Lasers

Helena Jelínková, Czech Technical University in Prague (Czech Republic)

$6 \quad$ Visible and UV Lasers I: Joint Session with Conferences 7580 and 7582

Norman Hodgson, Coherent, Inc. (United States)

Dahv A. V. Kliner, JDSU (United States)

Peter E. Powers, University of Dayton (United States)

$7 \quad$ Visible and UV Lasers II

W. Andrew Clarkson, University of Southampton (United Kingdom)

8 OPS Lasers 1

Helena Jelínková, Czech Technical University in Prague

(Czech Republic)

9 OPS Lasers II

Alan B. Petersen, Newport Spectra-Physics (United States)

10 Ceramic Materials and Thermal Management

W. Andrew Clarkson, University of Southampton (United Kingdom)

11 Ultrafast Lasers

Alan B. Petersen, Newport Spectra-Physics (United States)

12 Mid-Infrared Lasers I

Ramesh K. Shori, Naval Air Warfare Center (United States)

13 Mid-Infrared Lasers II

Ramesh K. Shori, Naval Air Warfare Center (United States) 
14 Q-switched Lasers

Norman Hodgson, Coherent, Inc. (United States)

15 Optics and Beam Delivery

David H. Titterton, Defence Science and Technology Laboratory

(United Kingdom)

16 Applications

Hans-Dieter Hoffmann, Fraunhofer-Institut für Lasertechnik (Germany) 
Downloaded From: https://www.spiedigitallibrary.org/conference-proceedings-of-spie on 26 Apr 2023

Terms of Use: https://www.spiedigitallibrary.org/terms-of-use 